\title{
DECOMPOSITION OF A $k$-COVECTOR WITH RESPECT TO A VECTOR AND COMPUTING ITS COMASS
}

\author{
DOAN THE HIEU
}

\section{Introduction}

A smooth differential form $\Phi$ on a Riemannian manifold which is closed and has comass one is called a calibration. Corresponding to a calibration is a geometry of minimal surfaces (cf. $[\mathrm{H}],[\mathrm{HL}]$ ).

The constant coefficient calibrations have been studied deeply by R. Harvey, B. Lawson, F. Morgan, J. Dadok ... Many beautiful constant coefficient calibrations and corresponding geometries were constructed in the nice paper of R. Harvey and B. Lawson [HL], for example Special Lagrangian, Associative, Coassociative, Cayley calibrations ... Computing the comass of a $k$-differential form is quite difficult, even in the simplest cases, the cases of $k$-covectors viewed as paralell differential forms. The known calibrations are not much, especially the calibrations of high degree. The such well-known calibrations are only Complex Line, Special Lagrangian, power of Kähler forms (see [DHM], [HL]).

The Associative and Coassociative calibrations (see [HL]) on $\boldsymbol{R}^{7}$ have many beautiful properties, and between them there is a relationship

$$
* \varphi=\psi,
$$

where $\varphi$ is Associative calibration, and $\psi$ is Coassociative calibration on $\boldsymbol{R}^{7}$.

Moreover,

and hence

$$
\varphi(\eta)^{2}+\bar{\psi}(\eta)^{2}=1 \quad \text { for all } \eta \in G\left(3, \boldsymbol{R}^{7}\right)
$$

$$
G(\varphi)=G_{0}(\bar{\psi})
$$

This paper gives a method to compute the comass of some classes of $k$ covectors, describes the set of all 3-covectors have comass one on $\boldsymbol{R}^{8}$, whose faces contain a $S L A G$ face (this set is denoted by $F^{*}(S L A G)$ ), and constructs new calibrations on $\boldsymbol{R}^{4 n-1}$ : General Associative and General Coassociative calibrations. The method bases on the decomposition of a covector $\Phi$ with respect to a vector $e \in \operatorname{span} \Phi^{*}$,

where $\varphi \in \bigwedge^{k-1}\left(e^{\perp}\right) ; \psi \in \bigwedge^{k}\left(e^{\perp}\right)$.

$$
\Phi=e^{*} \wedge \varphi+\psi
$$

Received September 16, 1997. 
Theorem 2.4 shows that

$$
\|\Phi\|^{*}=\max _{\eta \in G\left(k-1, e^{\perp}\right)} \sqrt{\varphi(\eta)^{2}+\bar{\psi}(\eta)^{2}}=A,
$$

and

$$
G(\Phi)=\left\{(\cos \alpha e+\sin \alpha f) \wedge \eta / \eta \in G\left(k-1, e^{\perp}\right)\right\}
$$

where

i) $\varphi(\eta)^{2}+\bar{\psi}(\eta)^{2}=A^{2}$

ii) $f=\frac{\bar{\psi}(\eta)}{\|\bar{\psi}(\eta)\|}$

iii) $\cos \alpha=\frac{\varphi(\eta)}{A}, \sin \alpha=\frac{\|\bar{\psi}(\eta)\|}{A}$.

By using this theorem and Theorems 6.11 and 6.16 in [HL], we prove that $\Phi_{G . A S S O C}$ and $\Phi_{G . C O A S S O C}$ are calibrations and the relationship $* \Phi_{G . A S S O C}=$ $\Phi_{G . C O A S S O C}$ holds.

\section{Decomposition of a covector with respect to a vector}

Let $\Phi$ be a $k$-covector on $\boldsymbol{R}^{n}(k<n)$, and suppose that $\left\{e_{1}, e_{2}, \ldots, e_{n}\right\}$ is an orthonormal basis of $\boldsymbol{R}^{n}$. Denote

$$
\begin{gathered}
\left.\Phi_{e_{i}}=e_{i}\right\lrcorner \Phi, \quad i=1,2, \ldots, n, \\
\text { (i.e. } \left.\quad \Phi_{e_{i}}(\eta)=\Phi\left(e_{i} \wedge \eta\right)\right),
\end{gathered}
$$

and

$$
\begin{aligned}
& \bar{\Phi}=\left(\Phi_{e_{1}}, \Phi_{e_{2}}, \ldots, \Phi_{e_{n}}\right), \\
& \text { (i.e. } \left.\quad \bar{\Phi}(\eta)=\sum \Phi_{e_{i}}(\eta) e_{i}\right) .
\end{aligned}
$$

Remark. Each $\Phi_{e_{i}}$ is a $(k-1)$-covector on $e_{i}{ }^{\perp}$, where $e_{i}{ }^{\perp}$ denote the subspace of $\boldsymbol{R}^{n}$ containing all vectors orthogonal to $e_{i}$.

LEMMA 2.1. 1) $\bar{\Phi}$ is a linear mapping from $\bigwedge_{-1}^{k-1}\left(\boldsymbol{R}^{n}\right)$ to $\boldsymbol{R}^{n}$.

2) $\Phi(x \wedge \eta)=\langle x, \bar{\Phi}(\eta)\rangle$, for all $x \in \boldsymbol{R}^{n} ; \eta \in \bigwedge^{k-1}\left(\boldsymbol{R}^{n}\right)$.

3) $\bar{\Phi}(\eta) \in \operatorname{span}(\eta)^{\perp}$, for all $\eta \in \bigwedge^{k-1}\left(\boldsymbol{R}^{n}\right)$, i.e. $\bar{\Phi}(\eta)$ is orthogonal to $\operatorname{span}(\eta)$.

Proof. 1) $\bar{\Phi}$ is linear since each $\Phi_{e_{i}}$ is linear.

2) Suppose that $x=\sum x_{i} e_{i}$, we have

$$
\begin{aligned}
\Phi\left(\sum x_{i} e_{i} \wedge \eta\right) & =\sum x_{i} \Phi\left(e_{i} \wedge \eta\right)=\sum x_{i} \Phi_{e_{i}}(\eta) \\
& =\langle x, \bar{\Phi}(\eta)\rangle .
\end{aligned}
$$


3) For each $x \in \operatorname{span}(\eta)$, we have

$$
\langle x, \bar{\Phi}(\eta)\rangle=\Phi(x \wedge \eta)=\Phi(0)=0 .
$$

Thus $\bar{\Phi}(\eta) \perp x$.

The proof of the lemma is completed.

As usual, we define the norm of $\bar{\Phi}$ (denote $\|\bar{\Phi}\|$ ) is the maximum value of $\|\bar{\Phi}\|$ attain on $G\left(k-1, \boldsymbol{R}^{n}\right), G(\bar{\Phi})$ is the set of all $\eta \in G\left(k-1, \boldsymbol{R}^{n}\right)$, where $\|\bar{\Phi}\|$ attains its maximum, and $G_{0}(\bar{\Phi})$ is the set of all $\eta \in G\left(k-1, \boldsymbol{R}^{n}\right)$ such that $\|\bar{\Phi}(\eta)\|=0$.

$$
\begin{gathered}
\|\bar{\Phi}\|=\max _{\eta \in G\left(k-1, R^{n}\right)}\|\bar{\Phi}(\eta)\|, \\
G(\bar{\Phi})=\left\{\eta \in G\left(k-1, R^{n}\right) /\|\bar{\Phi}(\eta)\|=\|\bar{\Phi}\|\right\}, \\
G_{0}(\bar{\Phi})=\left\{\eta \in G\left(k-1, R^{n}\right) /\|\bar{\Phi}(\eta)\|=0\right\} .
\end{gathered}
$$

LEMMA 2.2. 1) $\|\Phi\|^{*}=\|\bar{\Phi}\|$.

2) If $\|\Phi\|^{*}=1$, then $G(\Phi)=\{\bar{\Phi}(\eta) \wedge \eta / \eta \in G(\bar{\Phi})\}$.

Proof. 1) For each $\xi \in G\left(k, \boldsymbol{R}^{n}\right)$, let $e$ be a unit vector of $\operatorname{span}(\xi)$, thus $\xi=e \wedge \eta$, where $\eta \in G\left(k-1, R^{n}\right)$. Then

$$
\begin{aligned}
\Phi(\xi) & =\Phi(e \wedge \eta)=\langle e, \bar{\Phi}(\eta)\rangle \\
& \leq\|e\| \cdot\|\bar{\Phi}(\eta)\|=\|\bar{\Phi}(\eta)\| .
\end{aligned}
$$

Thus,

$$
\|\Phi\|^{*} \leq\|\bar{\Phi}\| .
$$

Now suppose $\eta \in G(\bar{\Phi})$. Let $e=\bar{\Phi}(\eta) /\|\bar{\Phi}(\eta)\|$, and $\xi=e \wedge \eta$. Then

$$
\begin{aligned}
\Phi(\xi) & =\Phi(e \wedge \eta)=\frac{1}{\|\bar{\Phi}(\eta)\|}\langle\bar{\Phi}(\eta), \bar{\Phi}(\eta)\rangle \\
& =\|\bar{\Phi}(\eta)\|=\|\bar{\Phi}\| .
\end{aligned}
$$

Thus,

$$
\|\Phi\|^{*} \geq\|\bar{\Phi}\|
$$

$(*)$ and $(* *)$ show that

$$
\|\Phi\|^{*}=\|\bar{\Phi}\| .
$$

2) The proof of the second part is clear. 
Suppose $\Phi$ is a $k$-covector on $\boldsymbol{R}^{n}$ with $\operatorname{span}(\Phi)^{*}=\boldsymbol{R}^{n}, \quad\left(\operatorname{span}(\Phi)^{*}=\right.$ $\left.\left.\left\{v \in \boldsymbol{R}^{n} / v\right\lrcorner \Phi=0\right\}^{\perp}\right)$. Let $e$ be a unit vector on $\boldsymbol{R}^{n}$, and

$$
\begin{aligned}
& \varphi=e\lrcorner \Phi, \\
& \psi=\Phi-e^{*} \wedge \varphi .
\end{aligned}
$$

We have the decomposition of $\Phi$ with respect to $e$

$$
\Phi=e^{*} \wedge \varphi+\psi
$$

where $\varphi$ and $\psi$ are respectively $(k-1)$-covector and $k$-covector on $e^{\perp}$.

The following lemma gives a relationship between $\|\Phi\|^{*},\|\varphi\|^{*}$ and $\|\psi\|^{*}$.

LEMMA 2.3.

$$
\max \left\{\|\varphi\|^{*},\|\psi\|^{*}\right\} \leq\|\Phi\|^{*} \leq \sqrt{\|\varphi\|^{* 2}+\|\psi\|^{* 2}} .
$$

Proof. Suppose $\Phi$ has the decomposition with respect to $e$

$$
\Phi=e^{*} \wedge \varphi+\psi
$$

Let $\eta \in G(\varphi)$ and let $\xi=e \wedge \eta$. Then

$$
\Phi(\xi)=e^{*} \wedge \varphi(\xi)+\psi(\xi)=\left(e^{*} \wedge \varphi\right)(\xi)=\varphi(\eta)=\|\varphi\|^{*} .
$$

Thus,

$$
\|\Phi\|^{*} \geq\|\varphi\|^{*}
$$

Let $\xi \in G(\psi)$, then $\left(e^{*} \wedge \varphi\right)(\xi)=0$, and hence

$$
\Phi(\xi)=\psi(\xi)=\|\psi\|^{*}
$$

Thus,

$$
\|\Phi\|^{*} \geq\|\psi\|^{*}
$$

$(*)$ and $(* *)$ prove the first inequality.

To prove the second inequality, we use the canonical form of a simple vector with respect to a subspace (see [HL], Lemma 7.5).

Let $\xi \in \bigwedge^{k}\left(\boldsymbol{R}^{n}\right)$, then $\xi$ has the canonical form with respect to the subspace $\operatorname{span}(e)=\{r . e / r \in \boldsymbol{R}\}$

$$
\xi=(\cos \alpha e+\sin \alpha f) \wedge \eta,
$$

where $e, f$ are orthonormal vectors, $\eta \in G\left(k-1, e^{\perp}\right) ; e \in \operatorname{span}(\eta)^{\perp} ; f \in \operatorname{span}(\eta)^{\perp}$. Then

$$
\begin{aligned}
\Phi(\eta) & =\cos \alpha \varphi(\eta)+\sin \alpha \psi(f \wedge \eta) \\
& \leq \sqrt{\cos ^{2} \alpha+\sin ^{2} \alpha} \cdot \sqrt{\varphi(\eta)^{2}+\psi(f \wedge \eta)^{2}} \\
& \leq \sqrt{\|\varphi\|^{* 2}+\|\psi\|^{* 2}} .
\end{aligned}
$$

The proof of the Lemma 2.3 is completed. 
More exactly, we have the following theorem.

THEOREM 2.4. 1) $\|\Phi\|^{*}=\max _{\eta \in G\left(k-1, e^{\perp}\right)}\left\{\sqrt{\varphi(\eta)^{2}+\bar{\psi}(\eta)^{2}}\right\}=A$.

2) $G(\Phi)=\left\{(\cos \alpha e+\sin \alpha f) \wedge \eta / \eta \in G\left(k-1, e^{\perp}\right)\right\}$, where

i) $\varphi(\eta)^{2}+\bar{\psi}(\eta)^{2}=A^{2}$

ii) $f=\frac{\bar{\psi}(\eta)}{\|\bar{\psi}(\eta)\|}$

iii) $\cos \alpha=\frac{\varphi(\eta)}{A}, \quad \sin \alpha=\frac{\|\bar{\psi}(\eta)\|}{A}$.

Proof. 1) By the virtue of the proof of Lemma 2.3, we imply that

$$
\|\Phi\|^{*} \leq \max \left\{\sqrt{\varphi(\eta)^{2}+\bar{\psi}(\eta)^{2}}\right\}=A .
$$

Now suppose the equality $\sqrt{\varphi(\eta)^{2}+\bar{\psi}(\eta)^{2}}=A$ holds for some $\eta \in G\left(k-1, e^{\perp}\right)$.

Let $f=\bar{\psi}(\eta) /\|\bar{\psi}(\eta)\| ; \cos \alpha=\varphi(\eta) / A ; \sin \alpha=\|\bar{\psi}(\eta)\| / A$, and

$$
\xi=(\cos \alpha e+\sin \alpha f) \wedge \eta,
$$

then

$$
\begin{aligned}
\Phi(\xi) & =\cos \alpha \varphi(\eta)+\sin \alpha\|\bar{\psi}(\eta)\| \\
& =\frac{\varphi(\eta)^{2}+\bar{\psi}(\eta)^{2}}{A}=\frac{A^{2}}{A}=A .
\end{aligned}
$$

The first part is proved.

2) The proof of the second part is clear.

Now we can give a criterion which can be used to check the comass one of some classes of covectors, and construct new examples of calibrations.

Corollary 2.5. Suppose $\Phi$ has the decomposition with respect to e, $\|e\|=1$

$$
\Phi=e^{*} \wedge \varphi+\psi
$$

where $\|\varphi\|^{*}=\|\psi\|^{*}=1$. Then we have

1) $\|\Phi\|^{*}=1$ if and only if $\sqrt{\varphi(\eta)^{2}+\bar{\psi}(\eta)^{2}} \leq 1$ for all $\eta \in G\left(k-1, \boldsymbol{R}^{n}\right)$.

2) If $\|\Phi\|^{*}=1$, then

$$
G(\Phi)=\left\{\xi=(\cos \alpha e+\sin \alpha f) \wedge \eta / \eta \in G\left(k-1, e^{\perp}\right)\right\},
$$

where

i) $\varphi(\eta)^{2}+\bar{\psi}(\eta)^{2}=1$.

ii) $f=\frac{\bar{\psi}(\eta)}{\|\bar{\psi}(\eta)\|}$.

iii) $\cos \alpha=\varphi(\eta), \sin \alpha=|\bar{\psi}(\eta)|$. 

2.4 .

The proof of the corollary is implied directly from the proof of the Lemma

\section{III. $F^{*}(S L A G)$ on $\boldsymbol{R}^{8}$}

Denote $F^{*}(S L A G)$ be the set of all calibrations on $\boldsymbol{R}^{n}$, whose faces contain a Special Lagrangian face. The first cousin principle shows that such calibrations must be in the form

$$
\Phi(\lambda, a)=\Phi_{S L A G}+\lambda\left(e_{14}^{*}+e_{25}^{*}+e_{36}^{*}\right) \wedge u^{*}+a \cdot v^{*} \wedge e_{78}^{*}
$$

where $u$ is a unit vector in $\operatorname{span}\left\{e_{7}, e_{8}\right\}$ and $v$ is a unit vector in $\operatorname{span}\left\{e_{1}, e_{2}, \ldots, e_{6}\right\}$. Here we used the notation $e_{i j k}^{*}$ instead of $e_{i}^{*} \wedge e_{J}^{*} \wedge e_{k}^{*}$. By using the action of $S O(2)$ on $\operatorname{span}\left\{e_{7}, e_{8}\right\}$, we can assume that $u=e_{7}$ and by using the action of $S U(3)$ on $\operatorname{span}\left\{e_{1}, e_{2}, \ldots, e_{6}\right\}$, we can assume that $v=e_{1}$.

LEMMA 3.1. Let $\Phi \in \bigwedge^{3}\left(\boldsymbol{R}^{8}\right)^{*}$ be in the form

$$
\Phi(\lambda, a)=\Phi_{S L A G}+\lambda\left(e_{14}^{*}+e_{25}^{*}+e_{36}^{*}\right) \wedge e_{7}^{*}+a e_{1}^{*} \wedge e_{78}^{*}
$$

then $\Phi(\lambda, a) \in F^{*}(S L A G)$ iff

$$
a^{2}+\lambda^{2} \leq 1
$$

Proof. We write $\Phi(\lambda, a)$ in the form

$$
\Phi(\lambda, a)=e_{8}^{*} \wedge\left(a e_{17}^{*}\right)+\Phi_{S L A G}+\lambda\left(e_{14}^{*}+e_{25}^{*}+e_{36}^{*}\right) \wedge e_{7}^{*}
$$

Then by virtue of the Theorem 2.4

$$
\|\Phi(\lambda, a)\|^{*}=1 \quad \text { iff } \quad\left\|a e_{17}^{*}(\eta)\right\|^{2}+\left\|\overline{\Phi_{S L A G}+\lambda\left(e_{14}^{*}+e_{25}^{*}+e_{36}^{*}\right) \wedge e_{7}^{*}}(\eta)\right\|^{2} \leq 1
$$

for all $\eta \in G\left(2, e_{8}^{\perp}\right) \subset \bigwedge^{2}\left(\operatorname{span}\left\{e_{1}, e_{2}, \ldots, e_{7}\right\}\right) \cong \bigwedge^{2}\left(\boldsymbol{R}^{7}\right)$.

Let $\psi=\Phi_{S L A G}+\lambda\left(e_{14}^{*}+e_{25}^{*}+e_{36}^{*}\right) \wedge e_{7}^{*} \in \bigwedge^{3}\left(\boldsymbol{R}^{7}\right)$, we have

$$
\begin{aligned}
& \psi_{e_{1}}=e_{23}^{*}-e_{56}^{*}+\lambda e_{47}^{*}, \\
& \psi_{e_{2}}=-e_{13}^{*}+e_{46}^{*}+\lambda e_{57}^{*}, \\
& \psi_{e_{3}}=e_{12}^{*}-e_{45}^{*}+\lambda e_{67}^{*}, \\
& \psi_{e_{4}}=-e_{26}^{*}+e_{35}^{*}-\lambda e_{17}^{*}, \\
& \psi_{e_{5}}=e_{16}^{*}-e_{34}^{*}-\lambda e_{27}^{*}, \\
& \psi_{e_{6}}=-e_{15}^{*}+e_{24}^{*}-\lambda e_{37}^{*}, \\
& \psi_{e_{7}}=\lambda\left(e_{14}^{*}+e_{25}^{*}+e_{36}^{*}\right) .
\end{aligned}
$$

(By simplicity we shall use the notation $e_{i j}$ for $e_{i j}^{*}(\eta)$ and $e_{i j} e_{k l}$ for $\left.e_{i j}^{*}(\eta) e_{k l}^{*}(\eta).\right) \quad$ We have 


$$
\begin{aligned}
a e_{17}^{*}(\eta)^{2}+\bar{\psi}(\eta)^{2}= & \left\|a e_{17}^{*}(\eta)\right\|^{2}+\sum\left\|\psi_{e_{i}}(\eta)\right\|^{2} \\
= & a^{2} e_{17}^{2}+e_{23}^{2}+e_{56}^{2}+\lambda^{2} e_{47}^{2}-e_{23} e_{56}+\lambda e_{23} e_{47}-\lambda e_{56} e_{47} \\
& +e_{13}^{2}+e_{46}^{2}+\lambda^{2} e_{57}^{2}-e_{13} e_{46}-\lambda e_{13} e_{57}+\lambda e_{46} e_{57} \\
& +e_{12}^{2}+e_{45}^{2}+\lambda^{2} e_{67}^{2}-e_{12} e_{45}+\lambda e_{12} e_{67}-\lambda e_{45} e_{67} \\
& +e_{26}^{2}+e_{35}^{2}+\lambda^{2} e_{17}^{2}-e_{26} e_{35}+\lambda e_{26} e_{17}-\lambda e_{35} e_{17} \\
& +e_{16}^{2}+e_{34}^{2}+\lambda^{2} e_{27}^{2}-e_{16} e_{34}-\lambda e_{16} e_{27}+\lambda e_{34} e_{27} \\
& +e_{15}^{2}+e_{24}^{2}+\lambda^{2} e_{37}^{2}-e_{15} e_{24}+\lambda e_{15} e_{37}-\lambda e_{24} e_{37} \\
& +\lambda^{2} e_{14}^{2}+\lambda^{2} e_{25}^{2}+\lambda^{2} e_{36}^{2}+\lambda^{2} e_{14} e_{25}+\lambda^{2} e_{14} e_{36}+\lambda^{2} e_{25} e_{36} \\
= & \left(e_{12}^{2}+e_{13}^{2}+e_{14}^{2}+e_{15}^{2}+e_{16}^{2}+e_{17}^{2}+e_{23}^{2}+e_{24}^{2}+e_{25}^{2}+e_{26}^{2}\right. \\
& +e_{27}^{2}+e_{34}^{2}+e_{35}^{2}+e_{36}^{2}+e_{37}^{2}+e_{45}^{2}+e_{46}^{2}+e_{47}^{2}+e_{56}^{2}+e_{57}^{2} \\
& +\left(\lambda^{2}-1\right)\left(e_{47}^{2}+e_{57}^{2}+e_{67}^{2}+e_{27}^{2}+e_{37}^{2}\right) \\
& +\left(\lambda^{2}-1\right)\left(e_{14}+e_{25}+e_{36}\right)^{2}+\left(a^{2}+\lambda^{2}-1\right) e_{17}^{2} \\
= & 1+\left(\lambda^{2}-1\right)\left(e_{27}^{2}+e_{37}^{2}+e_{47}^{2}+e_{57}^{2}+e_{67}^{2}\right) \\
& +\left(\lambda^{2}-1\right)\left(e_{14}+e_{25}+e_{36}\right)^{2}+\left(a^{2}+\lambda^{2}-1\right) e_{17}^{2} .
\end{aligned}
$$

- If $a^{2}+\lambda^{2}>1$, let $\eta \in G\left(2, \boldsymbol{R}^{7}\right)$ such that $\operatorname{span}(\eta)=\operatorname{span}\left\langle e_{1}, e_{7}\right\rangle$, then $\left\|a e_{17}^{*}(\eta)\right\|^{2}+\|\bar{\psi}(\eta)\|^{2}=a^{2}+\lambda^{2}>1$, and hence $\|\Phi\|>1$.

- If $a^{2}+\lambda^{2} \leq 1$, since

$$
\begin{aligned}
& \left(\lambda^{2}-1\right)\left(e_{47}^{2}+e_{57}^{2}+e_{67}^{2}+e_{27}^{2}+e_{37}^{2}\right) \leq 0, \\
& \left(\lambda^{2}-1\right)\left(e_{14}+e_{25}+e_{36}\right)^{2} \leq 0, \\
& \left(a^{2}+\lambda^{2}-1\right) e_{17}^{2} \leq 0,
\end{aligned}
$$

for all $\eta \in G\left(2, \boldsymbol{R}^{7}\right)$ we imply that $a e_{17}^{*}(\eta)^{2}+\bar{\psi}(\eta)^{2} \leq a^{2}+\lambda^{2} \leq 1$, and hence $\|\Phi\|^{*}=1$.

3.2. Classification of $F^{*}(S L A G)$ on $\boldsymbol{R}^{8}$

Now suppose $\|\Phi(\lambda, a)\|^{*}=1$, we have the folowing cases:

1. If $\lambda= \pm 1$, then $a=0$. $\Phi(\lambda, a)$ becomes to Associative-calibration and in this case $\Phi$ is an exposed calibration.

2. If $a= \pm 1$, then $\lambda=0 . \Phi(\lambda, a)$ is in the form

$$
\Phi=\Phi_{S L A G} \pm e_{178}^{*} \text {. }
$$

In this case $\Phi(\lambda)$ also an exposed calibration and $G(\Phi(\lambda, a))=G\left(\Phi_{S L A G}\right) \bigcup C P^{2}$. 
3. If $\lambda^{2}+a^{2}<1$, then $G(\Phi)=G\left(\Phi_{S L A G}\right)$. In this case $\Phi$ is not a maximal calibration and hence not exposed calibration.

4. If $\lambda^{2}+a^{2}=1(\lambda \neq 0$ and $a \neq 0), \eta$ must be a solution of the following system

$$
\left\{\begin{array}{l}
e_{27}^{*}=e_{37}^{*}=e_{47}^{*}=e_{57}^{*}=e_{67}^{*}=0 \\
e_{14}^{*}+e_{25}^{*}+e_{36}^{*}=0 . \quad \text { (Special Lagrangian condition) }
\end{array}\right.
$$

i) If $\operatorname{span}(\eta) \subset\left(e_{7}^{*}\right)^{\perp}$. Then $\xi \in G(\Phi(S L A G))$.

ii) If $\operatorname{span}(\eta) \not \subset\left(e_{7}^{*}\right)^{\perp}$. From the above system we imply that $\eta$ must be in the form

$$
e_{1} \wedge\left(a_{2} e_{2}+a_{3} e_{3}+a_{5} e_{5}+a_{6} e_{6}+a_{7} e_{7}\right)
$$

In this case, each $\Phi(\lambda, a)$ is a maximal calibration.

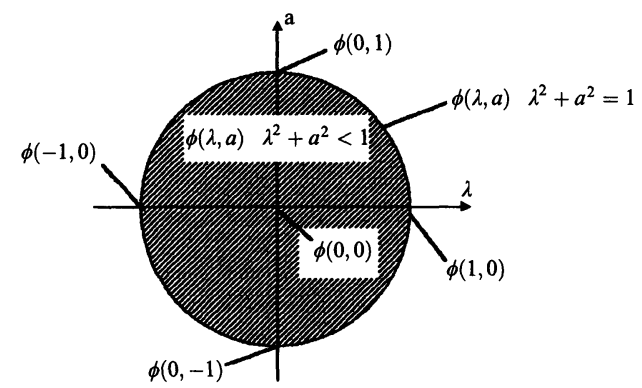

\section{General associative calibrations, general coassociative calibrations}

4.1. Strengthening of the Wirtinger inequality

First we iterate the result of the strengthening of Wirtinger inequality (Theorem 6.11 in [HL]) and another analog result (Remark 6.16 in [HL]), which are used to construct our new calibrations. $C^{n}$

THEOREM 4.1.1 (Theorem 6.11 in [HL]). For each (real) simple $2 p$-vector $\xi$ in

$$
\left|\Omega_{p}(\xi)\right|^{2}+\cdots+\sum_{|I|=2 k}^{\prime}\left|d Z^{I} \wedge \Omega_{p-k}(\xi)\right|^{2}+\cdots=|\xi|^{2} .
$$

If $2 p \leq n$ the last term on the left-hand side is $\sum_{|I|=2 p}^{\prime}\left|d Z^{I}(\xi)\right|^{2}$. If $2 p>n$ the left-hand side is $\sum_{|I|=2(n-p)}^{\prime}\left|d Z^{I} \wedge \Omega_{p-k}(\xi)\right|^{2}$.

THEOREM 4.1.2 (Remark 6.16 in [HL]). For each (real) simple $2 p+1$-vector $\xi$ in $C^{n}$

$$
\sum\left|d z_{J} \wedge \Omega_{p}(\xi)\right|^{2}+\cdots+\sum_{|I|=2 k+1}^{\prime}\left|d Z^{I} \wedge \Omega_{p-k}(\xi)\right|^{2}+\cdots=|\xi|^{2} .
$$


If $2 p+1 \leq n$ the last term on the left-hand side is $\sum_{|I|=2 p+1}^{\prime}\left|d Z^{I}(\xi)\right|^{2}$. If $2 p>n$ the left-hand side is $\sum_{|I|=2 p+1}^{\prime}\left|d Z^{I} \wedge \Omega_{2 p+1-n}(\xi)\right|^{2}$.

4.2. Some notes on associative and coassociative calibrations

As the next step, we give some notes on Associative and Coassociative calibrations on $\operatorname{Im}(O) \cong \boldsymbol{R}^{7}$. Theorem 1.4 in [HL] shows that the trilinear form $\Phi_{A S S O C}(x, y, z)=\langle x, y x\rangle$ on $\operatorname{Im}(O)$ is alternating and has comass one i.e a calibration (Associative calibration). Lemma 1.12 and Theorem 1.16 in [HL] show that the 4-form $\Phi_{C O A S S O C}(x, y, z, w)=1 / 2\langle x,[y, z, w]\rangle$ on $\operatorname{Im}(O)$ is alternating and has comass one i.e. a calibration (Coassociative calibration).

Let $e_{1}, e_{2}=i, e_{3}=j, e_{4}=k, e_{5}=e, e_{6}=i e, e_{7}=j e, e_{8}=k e$ denote the standard basis for the Octonions $O$. Let $e_{1}^{*}, e_{2}^{*}, \ldots, e_{8}^{*}$ denote the dual basis for $O^{*}$. By simplicity we use the notation $e_{p q r}^{*}$ for $e_{p}^{*} \wedge e_{q}^{*} \wedge e_{r}^{*}$. Then the form $\Phi_{A S S O C}$ can be expressed in terms of axis 3-planes as follows

$$
\begin{aligned}
\Phi_{A S S O C} & =e_{234}^{*}-e_{278}^{*}-e_{638}^{*}-e_{674}^{*}-e_{265}^{*}-e_{375}^{*}-e_{485}^{*} \\
& =e_{2}^{*} \wedge\left(e_{34}^{*}+e_{56}^{*}-e_{78}^{*}\right)+\left(e_{368}^{*}-e_{467}^{*}+e_{357}^{*}+e_{458}^{*}\right),
\end{aligned}
$$

and the form $\Phi_{\text {COASSOC }}$ can be expressed in terms axis 4-planes as follows

$$
\begin{aligned}
\Phi_{C O A S S O C} & =e_{5678}^{*}-e_{5634}^{*}-e_{5274}^{*}-e_{5238}^{*}+e_{3478}^{*}+e_{2468}^{*}+e_{2367}^{*} \\
& =e_{2}^{*} \wedge\left(e_{457}^{*}-e_{358}^{*}+e_{468}^{*}+e_{367}^{*}\right)+\left(e_{5678}^{*}-e_{3456}^{*}+e_{3478}^{*}\right) .
\end{aligned}
$$

Consider on $O$ the complex structure $J$ defined by

$$
e_{3}, e_{4}=J e_{3}, \quad e_{5}, e_{6}=J e_{5}, \quad e_{7}, e_{8}=-J e_{7}, \quad e_{1}, e_{2}=J e_{1} .
$$

On $\left(e_{1}, J e_{1}\right)^{\perp}$, let $\Omega$ denotes the Kähler form, $\Omega_{2}=1 / 2 \Omega^{2}$ and $\operatorname{Re}(d Z)$ denotes the Special Lagrangian form. Then $\Phi_{A S S O C}$ can be expressed as follows

$$
\Phi_{A S S O C}=J e_{1}^{*} \wedge(\Omega)+\operatorname{Re} d Z,
$$

and $\Phi_{\text {COASSOC }}$ can be expressed as follows

$$
\Phi_{\text {COASSOC }}=J e_{1}^{*} \wedge(\operatorname{Im} d Z)+\Omega_{2} .
$$

4.3. General associative calibrations

These are calibrations of degree $(2 n-1)$ on $\boldsymbol{R}^{4 n-1}$, and Associative calibration is the special case of them when $n=2$. Let $\left\{e_{1}, J e_{1}, e_{2}, J e_{2}, \ldots, e_{2 n}, J e_{2 n}\right\}$ be an orthnormal basis on $C^{2 n}$ correspond to the complex structure $J$. The subspace $\left(e_{n}, J e_{n}\right)^{\perp} \simeq C^{2 n-1}$ inherit the induced complex structure (we also denote by $J)$, and it is easy to see that $\left\{e_{1}, J e_{1}, e_{2}, J e_{2}, \ldots, e_{2 n-1}, J e_{2 n-1}\right\}$ is an orthonormal basis on it.

Let

$$
\operatorname{Re}\left(e^{i \theta} d Z\right)=\operatorname{Re}\left(e^{i \theta} d z_{1} \wedge d z_{2} \wedge \cdots \wedge d z_{2 n-1}\right)
$$

denotes the Special Lagrangian calibration (of degree $2(n-1)$ ) on $\left(e_{n}, J e_{n}\right)^{\perp}$ and 


$$
\Omega_{n-1}=\frac{1}{(n-1) !} \Omega^{n-1}
$$

where $\Omega$ is the Kähler form on $\left(e_{n}, J e_{n}\right)^{\perp}$. It is very easy to show that

$$
\begin{aligned}
& \operatorname{Re}\left(e^{i \theta} d Z\right)_{e_{i}}=\operatorname{Re}\left(e^{i \theta} d z_{1} \wedge d z_{2} \wedge \cdots \wedge \widehat{d z}_{l} \wedge \cdots \wedge d z_{2 n-1}\right), \\
& \operatorname{Re}\left(e^{i \theta} d Z\right)_{J e_{i}}=\operatorname{Im}\left(e^{i \theta} d z_{1} \wedge d z_{2} \wedge \cdots \wedge \widehat{d}_{\imath} \wedge \cdots \wedge d z_{2 n-1}\right),
\end{aligned}
$$

and hence for all $\eta \in G\left(2(n-1),\left(e_{n}, J e_{n}\right)^{\perp}\right)$

$$
\begin{aligned}
& \left|\overline{\operatorname{Re}\left(e^{i \theta} d Z\right)}(\eta)\right|^{2}=\sum\left|\operatorname{Re}\left(e^{i \theta} d z_{1} \wedge d z_{2} \wedge \cdots \wedge \widehat{d}_{l} \wedge \cdots \wedge d z_{2 n-1}\right)\right|^{2} \\
& +\sum\left|\operatorname{Im}\left(e^{i \theta} d z_{1} \wedge d z_{2} \wedge \cdots \wedge \widehat{d}_{l} \wedge \cdots \wedge d z_{2 n-1}\right)\right|^{2} \\
& =\sum\left|d z_{2} \wedge \cdots \wedge \widehat{d z}_{l} \wedge \cdots \wedge d z_{2 n}\right|^{2} \\
& =\sum_{|I|=2(n-1)}\left|d Z^{I}(\eta)\right|^{2}
\end{aligned}
$$

By virtue of the Theorem 3.1.1, we have

$$
\begin{aligned}
& \left|\Omega_{n-1}(\eta)\right|^{2}+\sum_{|I|=2(n-1)}\left|d Z^{I}(\eta)\right|^{2} \\
& \quad=\left|\Omega_{n-1}(\eta)\right|^{2}+|\overline{\operatorname{Re}(d Z)}(\eta)|^{2} \leq|\eta|^{2}=1,
\end{aligned}
$$

the equality holds if and only if

$$
\sum_{k=1}^{p-1} \sum_{|I|=2 k}^{\prime}\left|d Z^{I} \wedge \Omega_{p-k}(\eta)\right|^{2}=0 .
$$

More exactly $\eta$ is a solution of the following system of equations

$$
\begin{cases}\left|\operatorname{Re} d Z^{I} \wedge \Omega_{p-k}(\xi)\right|=0 & (|I|=2 k ; k=1,2, \ldots, p-1) \\ \left|\operatorname{Im} d Z^{I} \wedge \Omega_{p-k}(\xi)\right|=0 & (|I|=2 k ; k=1,2, \ldots, p-1)\end{cases}
$$

Let $\xi \in G\left(2(n-1), J e_{n} \oplus C^{2 n-1}\right)$. Suppose that $\xi$ has the canonical form with respect to the subspaces $\operatorname{span}\left(J e_{n}\right)$

$$
\xi=\left(\cos \alpha J e_{n}+\sin \alpha f\right) \wedge \eta .
$$
and

DEFINITION 4.3.3. $\xi$ is called G.ASSOCIATIVE if $\eta$ is a solution of (4.3.2.)

i) $f=\frac{\overline{\operatorname{Re} d Z}(\eta)}{\|\overline{\operatorname{Re} d Z}(\eta)\|}$

ii) $\cos \alpha=\Omega_{n-1}(\eta), \sin \alpha=|\overline{\operatorname{Re} d Z}(\eta)|$. 

by

Let $\Phi_{G . A S S O C}$ be the $(2 n-1)$-covector on $\operatorname{span}\left(J e_{n}\right) \oplus C^{2 n-1} \simeq R^{4 n-1}$ defined

$$
\Phi_{G . A S S O C(\theta)}=J e_{n} \wedge \Omega_{n-1}+\operatorname{Re}\left(e^{i \theta} d Z\right) .
$$

We have an $S^{1}$-family of G.ASSOC Geometries compatible with the given complex structure. Since these Geometries are equivalent under $S U(2 n-1)$, it will suffice to study the one associated to $\theta=0$.

THEOREM 4.3.4. The $(2 n-1)$-covector on $\operatorname{span}\left(J e_{n}\right) \oplus C^{2 n-1} \simeq \boldsymbol{R}^{4 n-1}$

$$
\Phi_{G . A S S O C}=J e_{n}^{*} \wedge \Omega_{n-1}+\operatorname{Re} d Z
$$

has comass one, i.e.

$$
\Phi_{G . A S S O C}(\xi) \leq|\xi| \text { for all } \xi \in G\left(2 n-1, R^{4 n-1}\right),
$$

and the equality holds iff $\xi$ is a G.ASSOCIATIVE, i.e.

$$
G\left(\Phi_{G . A S S O C}\right)=\{\xi / \xi \text { is a G.ASSOCIATIVE }\}
$$

Proof. The proof is implied directly by (4.3.1), (4.3.2) and Theorem 2.4.

4.4. General coassociative calibrations

These are calibrations of degree $2 n$ on $\boldsymbol{R}^{4 n-1}$, and Coassociative calibration is the special case of them when $n=2$.

Consider the Power of Kähler form of degree $2 n$ on $\left(e_{n}, J e_{n}\right)^{\perp}$, with the induced complex structure.

$$
\Omega_{n}=\frac{1}{n !} \Omega^{n},
$$

where $\Omega$ is the Kähler form on $\left(e_{n}, J e_{n}\right)^{\perp}$. Direct computation shows that

$$
\begin{aligned}
& \left(\Omega_{n}\right)_{e_{i}}=J e_{l}^{*} \wedge \Omega_{n-1}, \\
& \left(\Omega_{n}\right)_{J e_{l}}=e_{i}^{*} \wedge \Omega_{n-1},
\end{aligned}
$$

and hence for all $\eta \in G\left(2 n-1, \boldsymbol{R}^{4 n-1}\right)$

$$
\begin{aligned}
\left|\overline{\Omega_{n}}(\eta)\right|^{2} & =\sum\left|e_{i}^{*} \wedge \Omega_{n-1}(\eta)\right|^{2}+\sum\left|J e_{\imath}^{*} \wedge \Omega_{n-1}(\eta)\right|^{2} \\
& =\sum\left|d z_{l} \wedge \Omega_{n-1}(\eta)\right|^{2} .
\end{aligned}
$$

Let $d Z$ denote the Lagrangian form on $\left(e_{n}, J e_{n}\right)^{\perp}$. Then by virtue of the Theorem 4.3.2, we have

$$
\left|e^{i \theta} \operatorname{Im} d Z(\eta)\right|^{2}+\left|\overline{\Omega_{n}}(\eta)\right|^{2} \leq 1
$$

The equality holds if and only if
1) $|\operatorname{Re} d Z(\eta)|^{2}=0$
2) $\sum_{k=1}^{2(n-1)} \sum_{I=2 k+1}\left|d Z^{I} \wedge \Omega_{2 k+1}(\eta)\right|^{2}=0$. 
More exactly $\eta$ is a solution of the following system of equations

$$
\begin{cases}\operatorname{Re} d Z(\eta)=0 & \\ \operatorname{Re} d Z^{I} \wedge \Omega_{2 k+1}(\eta)=0 & (|I|=2 k+1 ; k=1,2, \ldots, 2(n-1)), \\ \operatorname{Im} d Z^{I} \wedge \Omega_{2 k+1}(\eta)=0 & (|I|=2 k+1 ; k=1,2, \ldots, 2(n-1)) .\end{cases}
$$

Let $\xi \in G\left(2 n, J e_{n} \oplus C^{2 n-1}\right)$. Suppose that $\xi$ has the canonical form with respect to the subspace $\operatorname{span}\left(J e_{n}\right)$

$$
\xi=\left(\cos \alpha J e_{n}+\sin \alpha f\right) \wedge \eta
$$

where $f \in C^{2 n-1}, \eta \in G\left(2 n-1, J e_{n} \oplus C^{2 n-1}\right)$.

DefintTion 4.4.3. $\xi$ is called G.COASSOCIATIVE if $\eta$ is a solution of (4.4.2) and
i) $f=\frac{\bar{\Omega}(\eta)}{\|\bar{\Omega}(\eta)\|}$.
ii) $\cos \alpha=\operatorname{Re} d Z(\eta) ; \sin \alpha=\left|\overline{\Omega_{n-1}}(\eta)\right|$.

Let $\Phi_{G . C O A S S O C}$ be the $2 n$-covectors on $\operatorname{span}\left(J e_{1}\right) \oplus C^{2 n-1} \simeq R^{4 n-1}$ defined by

$$
\Phi_{G . C O A S S O C}=J e_{n}^{*} \wedge \operatorname{Im}\left(e^{i \theta}\right) d Z-\Omega_{n}
$$

we have an $S^{1}$-family of G.COASSOC Geometries compatible with the given complex structure. Since these Geometries are equivalent under $S U(2 n-1)$, it will suffice to study the one associated to $\theta=0$.

THEOREM 4.4.4. The $2 n$-covector on $\operatorname{span}\left(J e_{n}\right) \oplus C^{2 n-1} \simeq R^{4 n-1}$

$$
\Phi_{G . C O A S S O C}=(-1)^{n} J e_{n}^{*} \wedge \operatorname{Im} d Z-\Omega_{n}
$$

has comass one, i.e.

$$
\Phi_{G . C O A S S O C}(\xi) \leq|\xi| \text { for all } \xi \in G\left(2 n, R^{4 n-1}\right),
$$

and the equality holds iff $\xi$ is G.COASSOCIATIVE.

Proof. The proof is implied directly by Theorem 2.4 and (4.4.1).

4.5. Relationship between G.ASSOC and G.COASSOC calibrations

In this section we give the first relationship between G.ASSOC and G.COASSOC calibrations. This relationship likes the first relationship between ASSOC and COASSOC calibrations

$$
* \Phi_{G . A S S O C}=\Phi_{G . C O A S S O C} \text {. }
$$

And in [Hi2] the second relationship

$$
\Phi_{G . A S S O C}(\eta)^{2}+\overline{\Phi_{G . C O A S S O C}}(\eta)^{2} \leq 1
$$

holds, for all $\eta \in G\left(2 n-1, R^{4 n-1}\right)$. 
If $n=2$ we the following equality holds for all $\eta \in G\left(3, R^{7}\right)$

$$
\Phi_{A S S O C}(\eta)^{2}+\overline{\Phi_{C O A S S O C}}(\eta)^{2}=1
$$

LEMMA 3.5.1. On $\boldsymbol{R}^{4 n-1}$

$$
* \Phi_{G . A S S O C}=\Phi_{G . C O A S S O C} .
$$

Proof. It is easily to see that: on $\boldsymbol{R}^{4 n-2}$

$$
\begin{gathered}
* \operatorname{Re} d Z=(-1)^{n-1} \operatorname{Im} d Z \\
* \Omega_{2 n}=\Omega_{2(n-1)}
\end{gathered}
$$

The proof is implied directly by 4.5 .2 and 4.5.3.

Acknowledgment. I would like to thank professor Dao Trong Thi for carefully reading the first draft of this paper and many valuable discussions. I also thank professor Frank Morgan for sending me some materials and the question about $F^{*}(S L A G)$ on $\boldsymbol{R}^{8}$.

\section{REFERENCES}

[DH] JiRI DADOK AND ReESe HaRveY, Calibratios on $\boldsymbol{R}^{6}$, Duke Math. J., 50 (1983), 1231-1243.

[DH1] JIRI DADOK AND REESE HARVEY, Calibrations and spinors, Rice University preprint (1989).

[DHM] JiRI DAdOK, Reese HaRvey AND Frank Morgan, Calibrations on $\boldsymbol{R}^{8}$, Trans. Amer. Math. Soc., 307 (1988), 1-40.

[H] REESE HARVEY, Calibrated geometries, Proceedings of the International Congress of Mathematics, Vol. 1,2 (Warsaw, 1983), PWN, Warsaw, 1984, 797-808.

[H1] Reese Harvey, Spinors and Calibrations, Academic Press, N. Y., 1990.

[HL] Reese HaRVey and H. Blaine Lawson, Calibrated geometries, Acta Math, 104 (1982), 47-157.

[HM] Reese HaRvey AND Frank Morgan, The comass ball in $\Lambda^{3}\left(\boldsymbol{R}^{6}\right)^{*}$, Indiana Math. J., 35 (1986), 145-156.

[Hil] DoAN The Hreu, On the comass norm of a 3-covector, Act. Math. Vietnam., 21 (1996), 349-367.

[Hi2] DOAN THE HIEU, On the calibrations invariant under transitive action, in preparation.

[M1] FRANK MORGAN, Area minimizing surfaces, faces of Grassmannians, and calibrations, Amer. Math. Monthly, to appear.

[M2] Frank Morgan, Calibrations and the size of Grassmann faces, Aequationes Math., 43 (1992), 1-13.

[Th1] Dao Trong Thi, Minımal sufaces in compact Lie groups, Uspekhı Mat. Nauk, 33 (1978), 163-164.

[Th2] Dao Trong ThI, Global minimal currents and surfaces in Riemannian manifolds, Acta Math. Vietnam., 10 (1985), 296-333.

HUE UNIVERSITY

32 LE LoI, HuE

VIETNAM 